\title{
Implementation of a novel enhanced recovery after surgery program in thoracoscopic bilateral bullectomy
}

\author{
Zhihua Guo, MD, Shuben Li, MD, PhD, Weiqiang Yin, MD, and \\ Jianxing He, MD, PhD, FACS, FRCS (Eng), Guangzhou, China
}

\footnotetext{
From the Department of Thoracic Surgery, First Affiliated Hospital of Guangzhou Medical University; Guangzhou Research Institute of Respiratory Disease; and Key cite of National Clinical Research Center for Respiratory Disease, Guangzhou, China

Disclosures: Authors have nothing to disclose with regard to commercial support.

Received for publication Sept 15, 2016; revisions received Oct 17, 2016; accepted for publication Oct 20, 2016; available ahead of print Dec 4, 2016.

Address for reprints: Jianxing He, MD, PhD, FACS, FRCS (Eng), Department of Thoracic Surgery, First Affiliated Hospital of Guangzhou Medical University, No. 151, Yanjiang Rd, Guangzhou 510120, China (E-mail: drjianxing.he@gmail.com).

J Thorac Cardiovasc Surg 2017; 153:e115-8

$0022-5223 / \$ 36.00$

Copyright (C) 2016 by The American Association for Thoracic Surgery

http://dx.doi.org/10.1016/j.jtcvs.2016.10.058
}

\section{Video clip is available online.}

Many hospitals worldwide have implemented enhanced recovery after surgery (ERAS) programs in thoracic departments. For bilateral bullectomy, video-assisted thoracic surgery (VATS) under intubated general anesthesia is the traditional ERAS approach. Here we present a novel ERAS approach using subxiphoid uniportal VATS under total intravenous anesthesia (TIVA) with a laryngeal mask (LM) to perform bilateral bullectomy without the use of postoperative chest drains. We believe patients under this novel approach may experience faster recovery times and may provide an alternative to the traditional ERAS approach.

\section{CLINICAL SUMMARY}

A 20-year-old man was admitted to our hospital due to chest pain and tightness with a known history of 2 separate episodes of primary spontaneous pneumothorax (PSP), 1 on the left and 1 on the right. Chest computed tomography scan showed a right-sided PSP, bilateral apical blebs, absence of extensive pleural adhesions, and absence of emphysematous changes. The patient had no history of surgical intervention for his PSP; thus, simultaneous bilateral thoracoscopic bullectomy was planned.

\section{TECHNIQUE}

The patient was placed in the supine position and TIVA with an LM, the details of which have been described in a previous report. ${ }^{1}$ The operation began with the patient spontaneously breathing oxygen via an LM. A single transverse

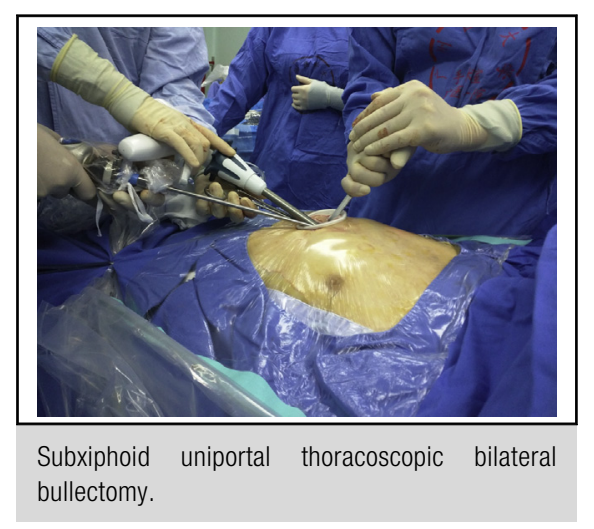

Central Message

The combined use of subxiphoid uniport, total intravenous anesthesia with a laryngeal mask, and omitting chest drains for thoracoscopic bilateral bullectomy is feasible.

See Editorial Commentary page e119.

3.0-cm skin incision was made below the xiphoid. A soft incision protector (Hakko Co, Ltd, Tokyo, Japan) was placed into the subcostal tunnel to obtain optimal exposure. After opening the right-sided pleural cavity, a natural collapse of the operative lung, comparable to that produced under intubated general anesthesia, occurred allowing maximal visualization of the lung (Figure 1, A). A 5-mm, $30^{\circ}$ rigid thoracoscopy (Stryker, Kalamazoo, Mich) was introduced. To reduce cough induced by thoracoscopic manipulation, $6 \mathrm{~mL} 2 \%$ lidocaine was sprayed on the lung surface (Figure 1, B). Subsequently, stapled-wedge resection was performed by using an articulating endostapler (Ethicon, Johnson \& Johnson, New Brunswick, NJ) (Figure 2, $A$ and $B$, and Video 1 ). While the patient was breathing spontaneously for the performance of the procedure on the right lung, when the left-sided pleural cavity was perforated, the consequent, bilateral iatrogenic pneumothorax may restrict lung expansion and impair spontaneous breathing. To ensure the patient sufficient levels of oxygenation, synchronized intermittent mandatory ventilation (SIMV) was delivered via LM for the remainder of the procedure. However, when SIMV was provided, bilateral lungs were inflated, which can affect surgical maneuvers. Therefore, after location of the blebs, SIMV was temporarily stopped while the left bullectomy was 

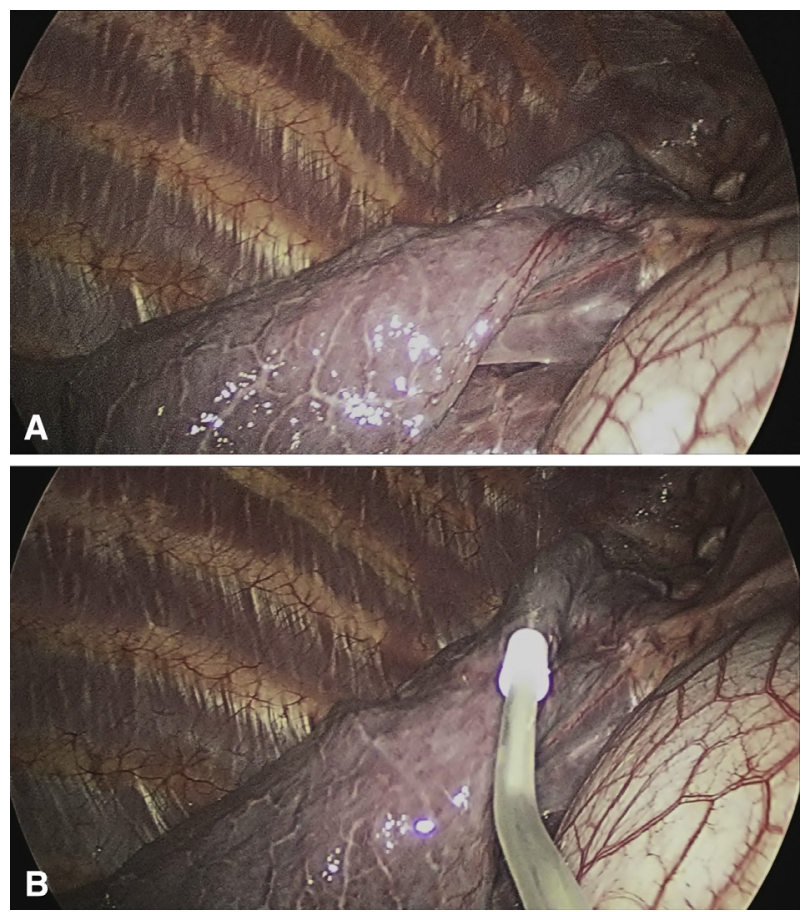

FIGURE 1. A, Intraoperative view demonstrating naturally collapsed lung during operation. B, $2 \%$ lidocaine was sprayed on the lung surface.

performed (Figure 2, $C$, Video 1). Thereafter, SIMV was immediately resumed and continued for the remainder of the operation. After insertion of 2 chest drains and wound closure, the bilateral lung was manually expanded via positive-pressure ventilation to check for air leaks and to remove the accumulated air from the pleural space. Chest drains were then removed and the thoracic cavity was quickly sealed (Figure 2,D).

The total operative duration was 50 minutes and estimated blood loss was minimal. The patient resumed his ambulation and diet 3 hours after the surgery. Postoperative pain was evaluated every 8 hours by means of a visual analog scale, which ranged from 0 (painless) to 10 (worst pain). The mean pain scores at rest were 3,2, and 1 on the day of surgery, and the first and second postoperative days, respectively. The patient was discharged uneventfully on the second postoperative day, and remained asymptomatic during 3 months of follow-up.

\section{DISCUSSION}

This case report shows our preliminary experience with a novel ERAS program in a patient with PSP undergoing subxiphoid uniportal thoracoscopic bilateral bullectomy under TIVA with an LM and omitting chest drains. This procedure avoids the use of intubated general anesthesia, the need to reposition the patient, and the use of chest drains. It may also further decrease postoperative intercostal neuralgia by bypassing the intercostal space and provide good cosmetic outcomes without chest scarring.
ERAS is a model of perioperative care. It has been developed via combining improved anesthetic technique, advanced minimally invasive surgery, and revision of conventional surgical care for elective surgery. The aim is to minimize surgical stress, prevent postoperative complications, and enhance recovery. Despite this, implementation of ERAS in daily practice is not yet widespread, as it challenges longstanding traditions. Thus, many barriers exist impeding implementation of such programs that need to be overcome to achieve successful widespread use.

Traditionally, intubated general anesthesia is mandatory for simultaneous bilateral thoracoscopic bullectomy. However, some complications after this anesthesia approach, such as intubation-related airway trauma, residual neuromuscular blockade, and postoperative nausea and vomiting, are not negligible and can be avoided by using TIVA with an LM. ${ }^{2}$ Our team is experienced with performing thoracic surgery under this anesthetic technique. ${ }^{1}$ Considering our favorable experiences, the requirement of exposure of both lungs, and the good physical condition of the young patient, we use this procedure using a subxiphoid uniportal approach under TIVA with an LM and without chest drains.

The major concern with the use of TIVA with an LM for bilateral bullectomy is hypoxia and hypercapnia, which may require conversion to intubated general anesthesia. During the procedure, the lowest intraoperative $\mathrm{SpO}_{2}$ level in the patient temporarily dropped to $89 \%$ and the peak $\mathrm{EtCO}_{2}$ rose to $68 \mathrm{~mm} \mathrm{Hg}$ after finishing the left-sided bullectomy. This patient tolerated the procedure well and hemodynamics were well maintained, showing that the use of LM to supplemental oxygen and assisted ventilation was sufficient to maintain satisfactory oxygenation and allowed us to provide deep sedation without compromising patient safety.

Currently, some scholars question the efficiency of thoracoscopic bullectomy alone due to the high recurrence rate. But the alternative to bullectomy alone (ie, the use of pleural abrasion after bullectomy for reducing pneumothorax recurrence) remains equally controversial. Combining our previous experience in bullectomy, our center considers that wedge resection alone to be sufficient in preventing PSP recurrence, especially in patients with isolated or limited bullae. ${ }^{1}$ Recent publications, including randomized controlled trials and meta-analyses, support our own experience, reporting bullectomy without pleural abrasion could achieve satisfactory results in preventing recurrent pneumothorax. ${ }^{3,4}$ Additionally, there exists a definite correlation between the extent of abrasion and development of complications, such as increased postoperative pleuritic pain and increased postoperative pleural drainage. These outcomes cause additional trauma and, in the case of this patient, would reduce the 

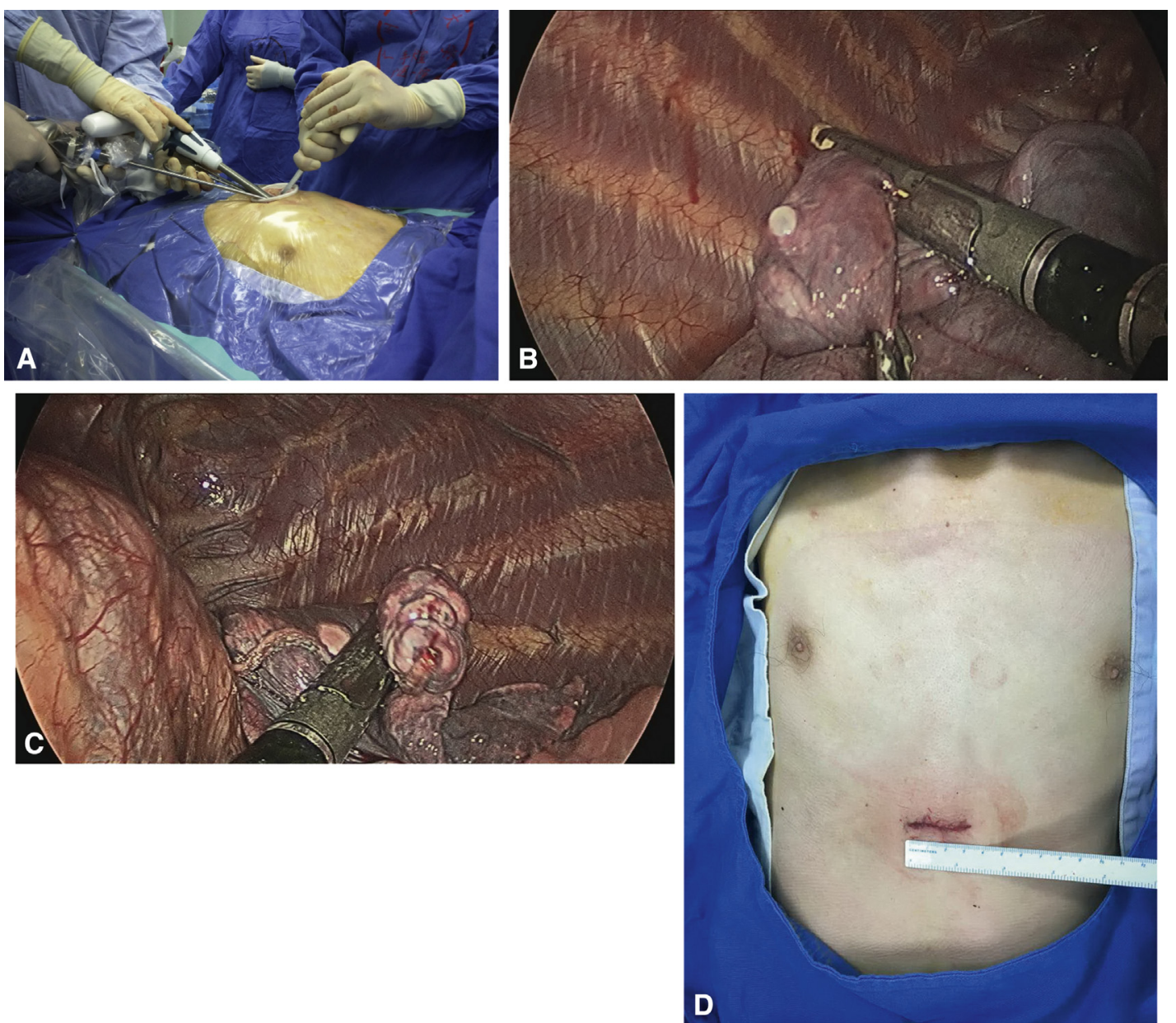

FIGURE 2. A, Three instruments were introduced through the subxiphoid uniport to perform the bullectomy. B, Intraoperative view of performing rightsided bullectomy. C, Intraoperative view of performing left-sided bullectomy. D, The postoperative wound.

potential advantages of omitting placement of chest drains.

Usually, chest drainage is a standard practice after VATS. However, it is associated with some adverse effects, such as enhanced pain at the incision site, discouraged coughing, and impaired mobility. In recent years,

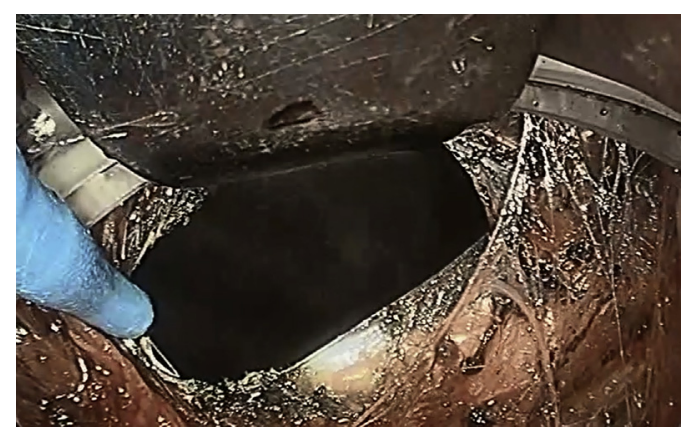

VIDEO 1. Subxiphoid uniportal thoracoscopic bilateral bullectomy under TIVA with an LM is shown. Video available at: http://www.jtcvsonline.org/ article/S0022-5223(16)31452-0/addons. the improvements in linear endostaplers have resulted in fewer air leaks and less bleeding after thoracoscopic wedge resection when appropriately used. These advances have led some surgeons to consider that chest drains may not be necessary in some patients undergoing thoracoscopic wedge resection. Watanabe et $\mathrm{al}^{5}$ demonstrated that omitting chest-drain placement after thoracoscopic wedge resection could be safe without increasing postoperative morbidity.

To minimize postsurgical pain, we modified the operative approach. Although uniportal VATS may avoid the need for multiple surgical incisions and therefore minimize surgical trauma, it is inevitably associated with chronic postoperative pain and chest wall numbness due to the damage to intercostal nerves. By combining the use of a subxiphoid incision and omission of chest drains, we believe we have decreased postoperative discomfort of the patient.

Although our patient had an uncomplicated recovery, he stayed in the hospital for 2 days as a precautionary measure because of the lack of local support services in his home 
county. Theoretically, he could have been discharged within 24 hours if district support services were available.

\section{CONCLUSIONS}

Subxiphoid uniportal thoracoscopic bilateral bullectomy under TIVA with an LM and without chest drains is a feasible technique in patients suitable for such an approach. We believe this treatment may contribute a less invasive alternative and potentially facilitate postoperative rapid recovery compared with traditional intercostal thoracoscopic surgery under intubated general anesthesia.

We thank Lindsey Hamblin for reviewing the manuscript.

\section{References}

1. Guo Z, Yin W, Wang W, Zhang J, Zhang X, Peng G, et al. Spontaneous ventilation anaesthesia: total intravenous anaesthesia with local anaesthesia or thoracic epidural anaesthesia for thoracoscopic bullectomy. Eur J Cardiothorac Surg. June 14, 2016 [Epub ahead of print].

2. Tsai TM, Chen JS. Nonintubated thoracoscopic surgery for pulmonary lesions in both lungs. J Thorac Cardiovasc Surg. 2012;144:e95-7.

3. Min X, Huang Y, Yang Y, Chen Y, Cui J, Wang C, et al. Mechanical pleurodesis does not reduce recurrence of spontaneous pneumothorax: a randomized trial. Ann Thorac Surg. 2014;98:1790-6.

4. Ling ZG, Wu YB, Ming MY, Cai SQ, Chen YQ. The effect of pleural abrasion on the treatment of primary spontaneous pneumothorax: a systematic review of randomized controlled trials. PLoS One. 2015;10:e0127857.

5. Watanabe A, Watanabe T, Ohsawa H, Mawatari T, Ichimiya Y, Takahashi N, et al. Avoiding chest tube placement after videoassisted thoracoscopic wedge resection of the lung. Eur J Cardiothorac Surg. 2004;25:872-6. 